\title{
Periodic Sequences of $p$-Class Tower Groups
}

\author{
Daniel C. Mayer \\ Dedicated to the memory of Emil Artin \\ Email: algebraic.number.theory@algebra
}

Received 31 March 2015; accepted 23 June 2015; published 30 June 2015

\begin{abstract}
Recent examples of periodic bifurcations in descendant trees of finite $p$-groups with $p \in\{2,3\}$ are used to show that the possible $p$-class tower groups $G$ of certain multiquadratic fields $K$ with $p$ class group of type $(2,2,2)$, resp. $(3,3)$, form periodic sequences in the descendant tree of the elementary Abelian root $C_{2}^{3}$, resp. $C_{3}^{2}$. The particular vertex of the periodic sequence which occurs as the $p$-class tower group $G$ of an assigned field $K$ is determined uniquely by the $p$-class number of a quadratic, resp. cubic, auxiliary field $k$, associated unambiguously to $K$. Consequently, the hard problem of identifying the $p$-class tower group $G$ is reduced to an easy computation of low degree arithmetical invariants.
\end{abstract}

\section{Keywords}

$p$-Class Field Towers, $p$-Principalization, $p$-Class Groups, Quadratic Fields, Multiquadratic Fields, Cubic Fields, Finite $p$-Groups, Parametrized Pc-Presentations, $p$-Group Generation Algorithm

\section{Introduction}

In this article, we establish class field theoretic applications of the purely group theoretic discovery of periodic bifurcations in descendant trees of finite $p$-groups, as described in our previous papers [1] [21] [22] (pp. 182-193) and [2] (§6.2.2), and summarized in section §2.

The infinite families of Galois groups of $p$-class field towers with $p \in\{2,3\}$ which are presented in sections $\S \S 4$ and 6 can be divided into different kinds. Either they form infinite periodic sequences of uniform step size 1, and thus of fixed coclass. These are the classical and well-known coclass sequences whose virtual periodicity has been proved independently by du Sautoy and by Eick and Leedham-Green (see [1], §7, pp. 167-168). Or they arise from infinite paths of directed edges in descendant trees whose vertices reveal periodic bifurcations (see [1], Thm.21.1, p. 182, [1], Thm.21.3, p. 185, and [2], Thm.6.4). Extensive finite parts of the latter have been constructed computationally with the aid of the $p$-group generation algorithm by Newman [3] and O'Brien [4] (see [1] [12]-[18]), but their indefinitely repeating periodic pattern has not been proven rigorously, so far. They can be of uniform step size 2 , as in $\S 4$, or of mixed alternating step sizes 1 and 2 , as in $\S 6$, whence their coclass increases unboundedly.

\section{Periodic Bifurcations in Trees of $p$-Groups}

For the specification of finite $p$-groups throughout this article, we use the identifiers of the SmallGroups 
database [5] [6] and of the ANUPQ-package [7] implemented in the computational algebra systems GAP [8] and MAGMA [9]-[11], as discussed in [1] (§9, pp. 168-169).

The first periodic bifurcations were discovered in August 2012 for the descendant trees of the 3-groups $Q=\langle 729,49\rangle$ and $U=\langle 729,54\rangle$ (see [1], §3, p. 163] and [1], Thm.21.3, p. 185), having abelian quotient invariants $(3,3)$, when we, in collaboration with Bush, conducted a search for Schur $\sigma$-groups as possible candidates for Galois groups $\mathrm{G}_{3}^{\infty}(K)=\operatorname{Gal}\left(\mathrm{F}_{3}^{\infty}(K) \mid K\right)$ of three-stage towers of 3-class fields over complex quadratic base fields $K=\mathbb{Q}(\sqrt{d})$ with $d \leq-9748$ and a certain type of 3-principalization [12] (Cor. 4.1.1, p. 775). The result in [12] (Thm. 4.1, p. 774) will be generalized to more principalization types and groups of higher nilpotency class in section $\S 6$.

Similar phenomena were found in May 2013 for the trees with roots $\langle 2187,168\rangle$ and $\langle 2187,181 \mid 191\rangle$ of type $(9,3)$ but have not been published yet, since we first have to present a classification of all metabelian 3groups with abelianization $(9,3)$.

At the beginning of 2014, we investigated the root $\langle 729,45\rangle$, which possesses an infinite balanced cover [2] (Dfn.6.1), and found periodic bifurcations in its decendant tree [2] (Thm.6.4).

In January 2015, we studied complex bicyclic biquadratic fields $K=\mathbb{Q}(\sqrt{-1}, \sqrt{d})$, called special Dirichlet fields by Hilbert [13], for whose 2-class tower groups $G_{2}^{\infty}(K)$ presentations had been given by Azizi, Zekhnini and Taous [14, Thm.2,(4)], provided the radicand $d$ exhibits a certain prime factorization which ensures a 2class group $\mathrm{Cl}_{2}(K)$ of type $(2,2,2)$.

In Section §4, we use the viewpoint of descendant trees of finite metabelian 2-groups and our discovery of periodic bifurcations in the tree with root $\langle 32,34\rangle$ [1] (Thm.21.1, p. 182) to prove a group theoretic restatement of the main result in the paper [14], which connects pairs $(m, n)$ of positive integer parameters with vertices of the descendant tree $\mathcal{T}(\langle 8,5\rangle)$ by means of an injective mapping $(m, n) \mapsto G_{m, n}$, as shown impressively in Figure 1.

\section{Pattern Recognition via Artin Transfers}

Let $p$ denote a prime number and suppose that $G$ is a finite $p$-group or an infinite pro-p group with finite abelianization $G / G^{\prime}$ of order $p^{v}$ with a positive integer exponent $v \geq 1$.

In this situation, there exist $v+1$ layers

$$
\operatorname{Lyr}_{n}(G):=\left\{G^{\prime} \leq H \leq G \mid(G: H)=p^{n}\right\} \text {, for } 0 \leq n \leq v,
$$

of intermediate normal subgroups $H \unlhd G$ between $G$ and its commutator subgroup $G^{\prime}$. For each of them, we denote by $T_{G, H}: G \rightarrow H / H^{\prime}$ the Artin transfer homomorphism from $G$ to $H$ [15]. In our recent papers [2] [3] [16], the components of the multiple-layered transfer target type (TTT) $\tau(G)=\tau_{0}(G) ; \ldots ; \tau_{v}(G)$ of $G$, resp. the multiple-layered transfer kernel type (TKT) $\varkappa(G)=\varkappa_{0}(G) ; \ldots ; \varkappa_{v}(G)$ of $G$, were defined by

$$
\tau_{n}(G):=\left(H / H^{\prime}\right)_{H \in \operatorname{Lyr}_{n}(G)} \text {, resp. } \varkappa_{n}(G):=\left(\operatorname{ker}\left(T_{G, H}\right)\right)_{H \in \operatorname{Lyr}_{n}(G)} \text {, for } 0 \leq n \leq v \text {. }
$$

The following information is known [16] to be crucial for identifying the metabelianization $G / G^{\prime \prime}$ of a p-class tower group $G$, but usually does not suffice to determine $G$ itself.

Definition 3.1 By the Artin pattern of $G$ we understand the pair

$$
\operatorname{AP}(G):=(\tau(G) ; \varkappa(G))
$$

onsisting of the multiple-layered TTT $\tau(G)$ and the multiple-layered TKT $\varkappa(G)$ of $G$.

If $G$ is the $p$-tower group of a number field $K$, then we put $\operatorname{AP}(K):=\operatorname{AP}(G)$ and speak about the Artin pattern of $K$.

As Emil Artin [15] pointed out in 1929 already, using a more classical terminology, the concepts transfer target type (TTT) and transfer kernel type (TKT) of a base field $K$, which we have now combined to the Artin pattern $(\tau(K) ; \varkappa(K))$ of $K$, require a non-abelian setting of unramified extensions of $K$. The reason is that the derived subgroup $H^{\prime}$ of an intermediate group $G^{\prime}<H<G$ between the $p$-tower group $G$ of $K$ and its commutator subgroup $G^{\prime}$ is an intermediate group between $G^{\prime}$ and the second derived subgroup $G^{\prime \prime}$. Therefore, the TTT $\tau(G)$ of the $p$-tower group $G=G_{p}^{\infty}(K)$ coincides with the TTT $\tau\left(G_{p}^{n}(K)\right)$ of any higher derived quotient $G_{p}^{n}(K) \simeq G / G^{(n)}$, for $n \geq 2$ but not for $n=1$, since $H / H^{\prime} \simeq\left(H / G^{(n)}\right) /\left(H^{\prime} / G^{(n)}\right)$, 


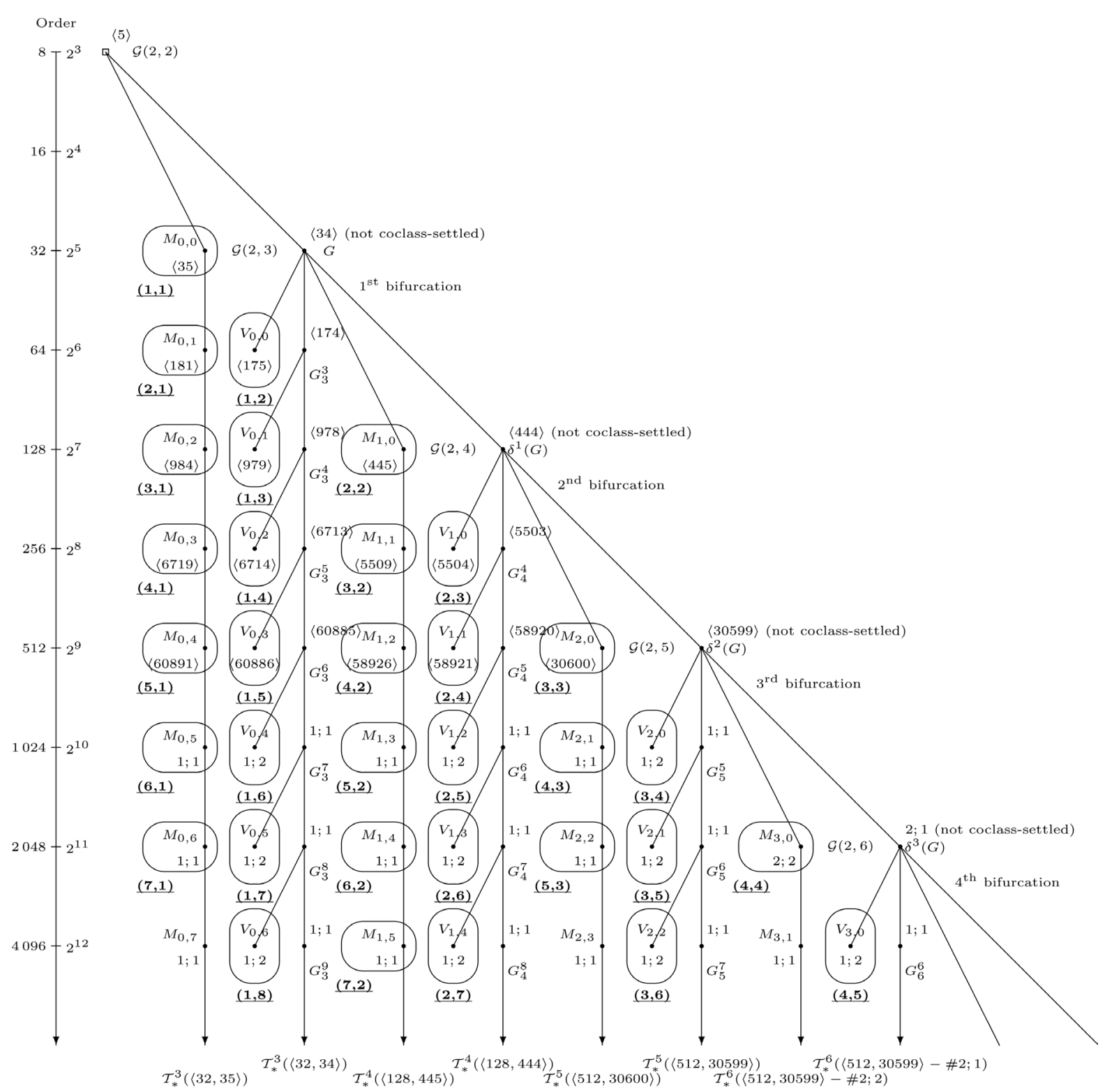

Figure 1. Pairs $(m, n)$ of parameters distributed over $\mathcal{T}_{*}(\langle 8,5\rangle)$.

according to the isomorphism theorem. Similarly, we have the coincidence of TKTs $\varkappa\left(G_{p}^{n}(K)\right)=\varkappa(G)$, for $n \geq 2$.

\section{Two-Stage Towers of 2-Class Fields}

As our first application of periodic bifurcations in trees of 2-groups, we present a family of biquadratic number fields $K$ with 2-class group $\mathrm{Cl}_{2}(K)$ of type $(2,2,2)$, discovered by Azizi, Zekhnini and Taous [14], whose 2-class tower groups $G=\mathrm{G}_{2}^{\infty}(K)$ are conjecturally distributed over infinitely many periodic coclass sequences, without gaps.

This claim is stronger than the statements in the following Theorem 4.1. The proof firstly consists of a group theoretic construction of all possible candidates for $G$, identified by their Artin pattern, up to nilpotency class $\operatorname{cl}(G) \leq 12$ and coclass $\operatorname{cc}(G) \leq 13$, thus having a maximal logarithmic order $\log _{2}(\operatorname{ord}(G)) \leq 25$. (The first part is independent of the actual realization of the possible groups $G$ as 2-tower groups of suitable fields $K$.) 
Secondly, evidence is provided of the realization of at least all those groups constructed in the first part whose logarithmic order does not exceed 11. The second part (see §5) is done by computing the Artin pattern of sufficiently many fields $K$ or by using more sophisticated ideas, presented in Theorem 4.1.

Remark 4.1 Generally, the first layer of the transfer kernel type $\varkappa_{1}(G)$ of $G$ will turn out to be a permutation [1] (Dfn.21.1, p. 182) of the seven planes in the 3-dimensional $\mathbb{F}_{2}$-vector space $G / G^{\prime} \simeq \mathrm{Cl}_{2}(K)$. We are going to use the notation of [1] (Thm.21.1 and Cor.21.1).

Theorem 4.1 Let $K=\mathbb{Q}(\sqrt{-1}, \sqrt{d})$ be a complex bicyclic biquadratic Dirichlet field with radicand $d=p_{1} p_{2} q$, where $p_{1} \equiv 1(\bmod 8), \quad p_{2} \equiv 5(\bmod 8)$ and $q \equiv 3(\bmod 4)$ are prime numbers such that $\left(\frac{p_{1}}{p_{2}}\right)=-1$ and $\left(\frac{p_{1}}{q}\right)=-1$.

Then the 2-class group $\mathrm{Cl}_{2}(K)$ of $K$ is of type $(2,2,2)$, the 2-class field tower of $K$ is metabelian (with exactly two stages), and the isomorphism type of the Galois group $G=\mathrm{G}_{2}^{\infty}(K)=\mathrm{Gal}\left(\mathrm{F}_{2}^{\infty}(K) \mid K\right)$ of the maximal unramified pro-2 extension $\mathrm{F}_{2}^{\infty}(K)$ of $K$ is characterized uniquely by the pair of positive integer parameters $(m, n)$ defined by the 2-class numbers $h_{2}\left(k_{1}\right)=2^{m+1}$ and $h_{2}\left(k_{2}\right)=2^{n}$ of the complex quadratic fields $k_{1}=\mathbb{Q}\left(\sqrt{-p_{1}}\right)$ and $k_{2}=\mathbb{Q}\left(\sqrt{-p_{2} q}\right)$.

The Legendre symbol $\left(\frac{p_{2}}{q}\right)$ decides whether $G$ is a descendant of $\langle 32,34\rangle$ or $\langle 32,35\rangle$ :

- $\left(\frac{p_{2}}{q}\right)=-1 \Leftrightarrow(m \geq) n=1 \Leftrightarrow$ the first layer TKT $\varkappa_{1}(G)$ is a permutation with five fixed points and a single 2-cycle $\Leftrightarrow G$ belongs to the mainline

$$
M_{0, k}:=\langle 32,35\rangle(-\# 1 ; 1)^{k} \text {, with } k=m-1 \geq 0,
$$

of the coclass tree $\mathcal{T}^{3}(\langle 32,35\rangle)$.

- $\left(\frac{p_{2}}{q}\right)=+1 \Leftrightarrow n>1 \Leftrightarrow$ the first layer TKT $\varkappa_{1}(G)$ is a permutation with a single fixed point and three 2-cycles $\Leftrightarrow G$ is a descendant of the group $\langle 32,34\rangle$, that is $G \in \mathcal{T}(\langle 32,34\rangle)$.

More precisely, in the second case the following equivalences hold in dependence on the parameters $m, n \leq \ell$, where $\ell \leq 11$ denotes a foregiven upper bound:

- $m \geq n \geq 2$ (with $n$ fixed) $\Leftrightarrow G$ belongs to the mainline

$$
M_{j+1, k}:=\langle 32,34\rangle(-\# 2 ; 1)^{j}-\# 2 ; 2(-\# 1 ; 1)^{k} \text {, with fixed } j=n-2
$$

and varying $k=m-n \geq 0$, of the coclass tree $\mathcal{T}^{n+2}\left(\langle 32,34\rangle(-\# 2 ; 1)^{n-2}-\# 2 ; 2\right)$.

- $n>m \geq 1$ (with $m$ fixed) $\Leftrightarrow G$ belongs to the unique periodic coclass sequence

$$
V_{j, k}:=\langle 32,34\rangle(-\# 2 ; 1)^{j}(-\# 1 ; 1)^{k}-\# 1 ; 2 \text {, with fixed } j=m-1
$$

and varying $k=n-m-1 \geq 0$, whose members possess a permutation as their first layer transfer kernel type, of the coclass tree $\mathcal{T}^{m+2}\left(\langle 32,34\rangle(-\# 2 ; 1)^{m-1}\right)$.

We add a corollary which gives the Artin pattern of the groups in Theorem 4.1, firstly, since it is interesting in its own right, and secondly, because we are going to use its proof as a starting point for the proof of Theorem 4.1.

Corollary 4.1 Under the assumptions of Theorem 4.1, the Artin pattern $\operatorname{AP}(G)=(\tau(G) ; \varkappa(G))$ of the 2tower group $G=\mathrm{G}_{2}^{\infty}(K)$ of the biquadratic field $K=\mathbb{Q}(\sqrt{-1}, \sqrt{d})$ is given as follows:

The ordered multi-layered transfer target type (TTT) $\tau(G)=\tau_{0} ; \tau_{1} ; \tau_{2} ; \tau_{3}$ of the Galois group $G$ is given by $\tau_{0}=\left(1^{3}\right), \quad \tau_{3}=(m, n)$, and 


$$
\begin{gathered}
\tau_{1}=\left\{\begin{array}{l}
(m+1,2),(2,1)^{2},\left(1^{3}\right)^{2},(2,1)^{2}, \text { if }\left(\frac{p_{2}}{q}\right)=-1, \\
(m+1, n+1),\left(1^{3}\right)^{6}, \text { else, }
\end{array}\right. \\
\tau_{2}=\left\{\begin{array}{l}
(m+1,1),(m, 2),(m+1,1),(2,1)^{4}, \text { if }\left(\frac{p_{2}}{q}\right)=-1, \\
(m+1, n),(m, n+1),(\max (m+1, n+1), \min (m, n)),\left(1^{3}\right)^{4}, \text { else. }
\end{array}\right.
\end{gathered}
$$

If we now denote by $N_{i}:=\operatorname{Norm}_{K_{i} \mid K}\left(\mathrm{Cl}_{2}\left(K_{i}\right)\right), 1 \leq i \leq 7$, the norm class groups of the seven unramified quadratic extensions $K_{i} \mid K$, then the ordered multi-layered transfer kernel type (TKT) $\varkappa_{(G)}=\varkappa_{0} ; \varkappa_{1} ; \varkappa_{2} ; \varkappa_{3}$ of the Galois group $G$ is given by $\varkappa_{0}=1, \varkappa_{2}=\left(0^{7}\right), \varkappa_{3}=(0)$, and

$$
\varkappa_{1}=\left\{\begin{array}{l}
\left(N_{1}, N_{2}, N_{3}, N_{5}, N_{4}, N_{6}, N_{7}\right), \text { if }\left(\frac{p_{2}}{q}\right)=-1, \\
\left(N_{1}, N_{3}, N_{2}, N_{5}, N_{4}, N_{7}, N_{6}\right), \text { else. }
\end{array}\right.
$$

Thus, $\varkappa_{1}$ is always a permutation of the norm class groups $N_{i}$. For $\left(\frac{p_{2}}{q}\right)=-1$ it contains five fixed points and a single 2-cycle, and otherwise it contains a single fixed point and three 2-cycles.

Proof. The underlying order of the 7 unramified quadratic, resp. bicyclic biquadratic, extensions of $K$ is taken from [14] (\$2.1, Thm.1, (3), (5)).

For the TTT we use logarithmic abelian type invariants as explained in [2] (§2). $\tau_{0}$ is taken from [14] (§2.2, Thm.2, (1)), $\tau_{1}, \tau_{2}$ from [14] (2.3, Thm.3, (1), (2)), and $\tau_{3}$ from [14] (\$2.2, Thm.2, (5)).

Concerning the TKT, $\varkappa_{0}$ is trivial, $\varkappa_{1}, \varkappa_{2}$ are taken from [14] (§2.3, Thm.3, (3)-(5)), and $\varkappa_{3}$ is total, due to the Hilbert/Artin/Furtwängler principal ideal theorem.

Proof. (Proof of Theorem 4.1)

Firstly, the equivalence $\left(\frac{p_{2}}{q}\right)=-1 \Leftrightarrow n=1$ is proved in [14] (3, Lem.5).

Next, we use the Artin pattern of $G$, as given in Corollary 4.1, to narrow down the possibilities for $G$. The possible class-2 quotients of $G$ are exactly the immediate descendants of the root $\langle 8,5\rangle$, that is, three vertices $\langle 16,11 \ldots 13\rangle$ of step size 1 , nine vertices $\langle 32,27 \ldots 35\rangle$ of step size 2 , and ten vertices $\langle 64,73 \ldots 82\rangle$ of step size 3. Among all descendants of $\langle 8,5\rangle$, the mainline vertices of the tree $\mathcal{T}(\langle 32,35\rangle)$ are characterized uniquely by the fact that their first layer TKT $\varkappa_{1}$ is a permutation with five fixed points and a single 2-cycle, and that their first layer TTT $\tau_{1}$ contains the unique polarized (i.e. parameter dependent) component $(m+1,2)$. Note that the mainline vertices of the tree $\mathcal{T}(\langle 32,31\rangle)$ reveal the same six stable (i.e. parameter independent) components $\left(\left(1^{3}\right)^{2},(2,1)^{4}\right)$ of the accumulated (unordered) first layer TTT $\tau_{1}$, but their first layer TKT $\varkappa_{1}$ contains three 2-cycles, similarly as for descendants of $\langle 32,34\rangle$. However, vertices of the complete descendant tree $\mathcal{T}(\langle 32,34\rangle)$ are characterized uniquely by six stable components $\left(\left(1^{3}\right)^{6}\right)$ of their first layer TTT $\tau_{1}$.

So far, we have been able to single out that $G$ must be a descendant of either $\langle 32,34\rangle$ or $\langle 32,35\rangle$, by means of Artin patterns, without knowing a presentation. Now, the parametrized presentation for the group $G=G_{m, n}$ in [14] (§2.2, Thm.2, (4)),

$$
G_{m, n}=\left\langle\rho, \sigma, \tau \mid \rho^{4}=\sigma^{2^{n+1}}=\tau^{2^{m+1}}=1, \rho^{2}=\sigma^{2^{n}}, \rho, \sigma=\sigma^{2}, \rho, \tau=\tau^{2}, \sigma, \tau=1\right\rangle,
$$

is used as input for a Magma program script [10] [11] which identifies a 2-group, given by generators and relations,

Group $\langle\rho, \sigma, \tau|$ relatorwordsin $\rho, \sigma, \tau\rangle$, with the aid of the following functions:

- CanIdentify Group() and Identify Group() if $|G| \leq 2^{8}$,

- Is In Small Group Database(), pQuotient(), Number Of Small Groups(), Small Group() and Is Isomorphic() if $|G|=2^{9}$, and 
- Generatep Groups(), resp. a recursive call of Descendants() (using Nuclear Rank() for the recursion), and Is Isomorphic() if $|G| \geq 2^{10}$.

The output of the Magma script is in perfect accordance with the pruned descendant tree $\mathcal{T}_{*}(\langle 8,5\rangle)$, as described in Theorem 21.1 and Corollary 21.1 of [1] (pp.182-183).

Finally, the class and coclass of $G$ are given in [14] (§2.2, Thm.2, (6)).

\section{Computational Results for Two-Stage Towers}

With the aid of the computational algebra system MAGMA [11], we have determined the pairs of parameters $(m, n)=(m(d), n(d))$, investigated in [14], for all 11753 square free radicands $d=p_{1} p_{2} q$ of the shape in Theorem 4.1 which occur in the range $0<d<2 \cdot 10^{6}$. As mentioned at the beginning of $\S 4$, the result supports the conjecture that the corresponding 2-tower groups $G_{m(d), n(d)}$ cover the pruned tree $\mathcal{T}_{*}(\langle 8,5\rangle)$ without gaps.

Recall that a pair $(m, n)$ contains information on the 2-class numbers of complex quadratic fields. So we have a reduction of hard problems for biquadratic fields to easy questions about quadratic fields.

By means of the following invariants, the statistical distribution $d \mapsto(m(d), n(d))$ of parameter pairs is visualized on the pruned descendant tree $\mathcal{T}_{*}(\langle 8,5\rangle)$, using the injective (and probably even bijective) mapping $(m, n) \mapsto G_{m, n}$. For each fixed individual pair $(m, n)$, we define its minimal radicand $M(m, n)$ as an absolute invariant:

$$
M(m, n):=\min \{d>0 \mid(m(d), n(d))=(m, n)\} .
$$

The purely group theoretic pruned descendant tree was constructed in [1] (§21.1, pp. 182-184), and was shown in [1] (\$10.4.1, Figure 7, p. 175), with vertices labelled by the standard identifiers in the SmallGroups Library [5] [6] or of the ANUPQ-package [7].

In Figure 1, a pair $(m, n)$ of parameters is placed adjacent to the corresponding vertex $G_{m, n}$ of the pruned descendant tree $\mathcal{T}_{*}(\langle 8,5\rangle)$. There are no overlaps, since the mapping $(m, n) \mapsto G_{m, n}$ is injective. Each vertex is additionally labelled with a formal identifier, as used in [1] (Cor.21.1).

In Figure 2, the minimal radicand $M(m, n)$ for which the adjacent vertex is realized as the corresponding group $G_{m, n}$, is shown underlined and with boldface font.

Vertices within the support of the distribution are surrounded by an oval. The oval is drawn in horizontal orientation for mainline vertices and in vertical orientation for vertices in other periodic coclass sequences.

\section{Three-Stage Towers of 3-Class Fields}

Our second discovery of periodic bifurcations in trees of 3-groups will now be applied to a family of quadratic number fields $K$ with 3-class group $\mathrm{Cl}_{3}(K)$ of type $(3,3)$, originally investigated by ourselves in [16]-[18], and extended by Boston, Bush and Hajir in [19]. The 3-class tower groups $G=G_{3}^{\infty}(K)$ of these fields are conjecturally distributed over six periodic sequences arising from repeated bifurcations (of the new kind which was unknown up to now), whereas it is proven that their metabelianizations populate six well-known periodic coclass sequences of fixed coclass 2 .

Theorem 6.1 Let $K=\mathbb{Q}(\sqrt{d})$ be a complex quadratic field with discriminant $d<0$, having a 3-class group $\mathrm{Cl}_{3}(K)$ of type $(3,3)$, such that its 3 -principalization in the four unramified cyclic cubic extensions $L_{1}, \ldots, L_{4}$ is given by one of the following two first layer TKTs

$$
\varkappa_{1}(K)=(1,1,2,2) \text { or }(3,1,2,2) \text {, }
$$

resp.

$$
\varkappa_{1}(K)=(2,2,3,4) \text { or }(2,3,3,4) .
$$

Further, let the integer $2 \leq \ell \leq 9$ denote a foregiven upper bound.

Then the 3-class field tower of $K$ is non-metabelian with exactly three stages, and the isomorphism type of the Galois group $G=\mathrm{G}_{3}^{\infty}(K)=\mathrm{Gal}\left(\mathrm{F}_{3}^{\infty}(K) \mid K\right)$ of the maximal unramified pro-3 extension $\mathrm{F}_{3}^{\infty}(K)$ of $K$ is characterized uniquely by the positive integer parameter $2 \leq u \leq \ell$ defined by the 3-class number $h_{3}\left(k_{0}\right)=3^{u}$ of the simply real non-Galois cubic subfield $k_{0}$ of the distinguished polarized extension $L$ among $L_{1}, \ldots, L_{4}$ (i.e., $L=L_{1}$, resp. $L=L_{2}$ ): 


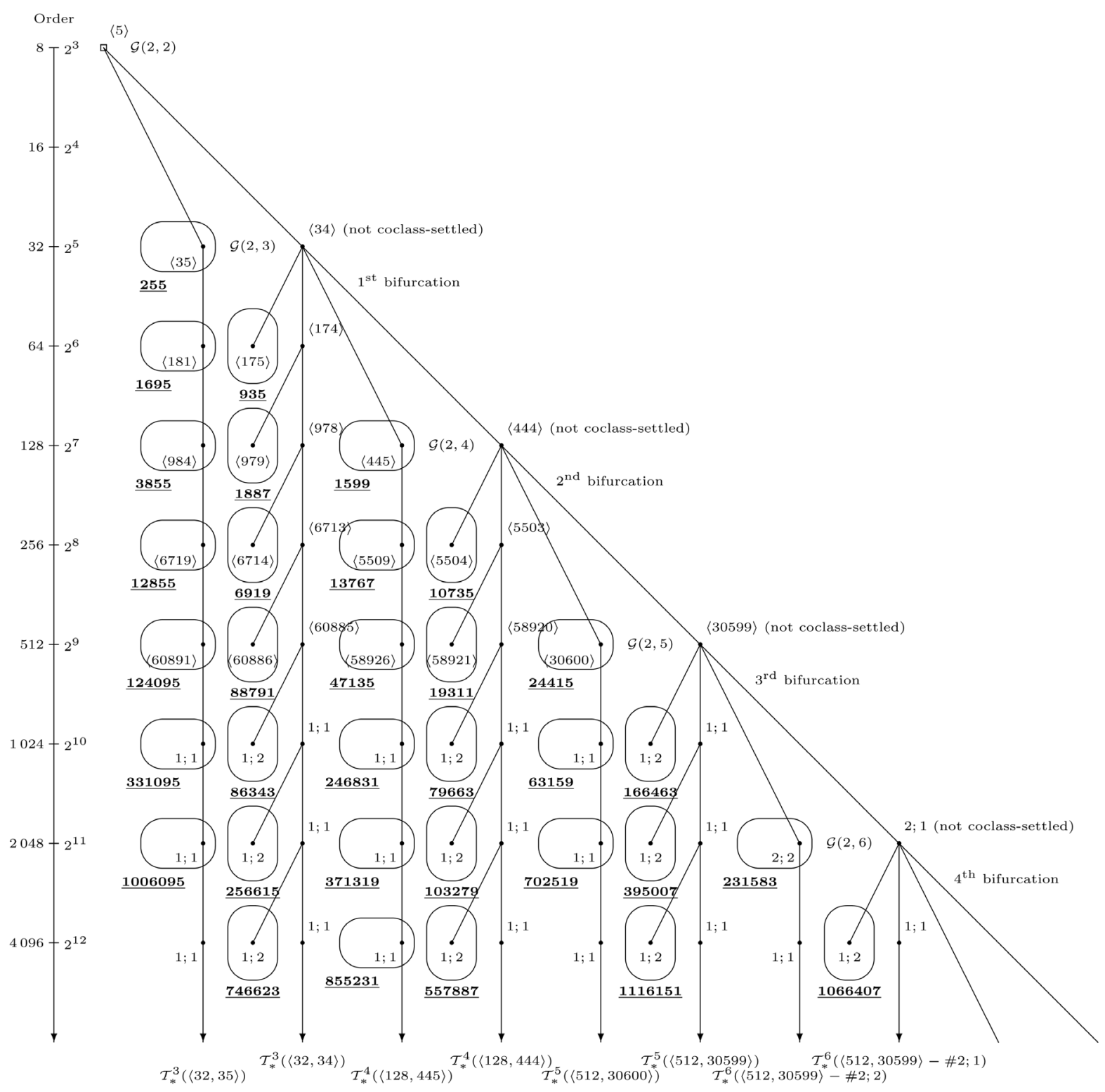

Figure 2. Minimal radicands $d$ distributed over $\mathcal{T}_{*}(\langle 8,5\rangle)$.

$$
\begin{aligned}
& G \simeq\langle 729,49\rangle(-\# 2 ; 1-\# 1 ; 1)^{j}-\# 2 ; 4 \text { or } 5 \mid 6 \text {, resp. } \\
& G \simeq\langle 729,54\rangle(-\# 2 ; 1-\# 1 ; 1)^{j}-\# 2 ; 2 \text { or } 4 \mid 6 \text {, with } j=u-2 .
\end{aligned}
$$

The metabelianization $G / G^{\prime \prime}$ of the Schur $\sigma$-group $G$, that is the Galois group $\mathrm{G}_{3}^{2}(K)=\operatorname{Gal}\left(\mathrm{F}_{3}^{2}(K) \mid K\right)$ of the maximal metabelian unramified 3-extension $\mathrm{F}_{3}^{2}(K)$ of $K$ is unbalanced and given by

$$
\begin{aligned}
& G / G^{\prime \prime} \simeq\langle 729,49\rangle(-\# 1 ; 1-\# 1 ; 1)^{k}-\# 1 ; 4 \text { or } 5 \mid 6, \text { resp. } \\
& G / G^{\prime \prime} \simeq\langle 729,54\rangle(-\# 1 ; 1-\# 1 ; 1)^{k}-\# 1 ; 2 \text { or } 4 \mid 6, \text { with } k=u-2 .
\end{aligned}
$$

Again, we first state a corollary whose proof will initialize the proof of Theorem 6.1.

Corollary 6.1 Under the assumptions of Theorem 6.1, the Artin pattern $\operatorname{AP}(G)=(\tau(G) ; \varkappa(G))$ of the 3tower group $G=\mathrm{G}_{3}^{\infty}(K)$ of the complex quadratic field $K=\mathbb{Q}(\sqrt{d})$ is given as follows: 
The ordered multi-layered transfer target type (TTT) $\tau(G)=\tau_{0} ; \tau_{1} ; \tau_{2}$ of the Galois group $G$ is given by $\tau_{0}=\left(1^{3}\right), \tau_{2}=(u, u, 1)$, and

$$
\tau_{1}=\left\{\begin{array}{l}
(u+1, u), 1^{3},(2,1)^{2}, \text { if } G \in \mathcal{T}(\langle 729,49\rangle) \\
(2,1),(u+1, u),(2,1)^{2}, \text { if } G \in \mathcal{T}(\langle 729,54\rangle)
\end{array}\right.
$$

If we now denote by $N_{i}:=\operatorname{Norm}_{L_{i} \mid K}\left(\mathrm{Cl}_{3}\left(L_{i}\right)\right), 1 \leq i \leq 4$, the norm class groups of the four unramified cyclic cubic extensions $L_{i} \mid K$, then the ordered multi-layered transfer kernel type (TKT) $\varkappa_{(G)}=\varkappa_{0} ; \varkappa_{1} ; \varkappa_{2}$ of the Galois group $G$ is given by $\varkappa_{0}=1, \varkappa_{2}=(0)$, and

$$
\varkappa_{1}=\left\{\begin{array}{l}
\left(N_{1}, N_{1}, N_{2}, N_{2}\right) \text { or }\left(N_{3}, N_{1}, N_{2}, N_{2}\right), \text { if } G \in \mathcal{T}(\langle 729,49\rangle), \\
\left(N_{2}, N_{2}, N_{3}, N_{4}\right) \text { or }\left(N_{2}, N_{3}, N_{3}, N_{4}\right), \text { if } G \in \mathcal{T}(\langle 729,54\rangle) .
\end{array}\right.
$$

Thus, $\varkappa_{1}$ is not a permutation of the norm class groups $N_{i}$. For $G \in \mathcal{T}(\langle 729,49\rangle)$ it contains a single or no fixed point and no 2-cycle, and for $G \in \mathcal{T}(\langle 729,54\rangle)$ it contains three or two fixed points and no 2-cycle.

Proof. First, we must establish the connection of the TTT of $G$ with the distinguished non-Galois simply real cubic field $k_{0}$. Anticipating the partial result of Theorem 6.1 that the metabelianization $G / G^{\prime \prime}$ of $G$ must be of coclass $r=2$, we can determine the 3-class numbers of all four non-Galois cubic subfields $k_{i}<L_{i}$ with the aid of Theorem 4.2 in [17] (p. 489): with respect to the normalization in this theorem, we have $h_{3}\left(k_{0}\right)=3^{u}=h_{3}\left(k_{1}\right)=3^{\frac{m-2}{2}}$ and uniformly $h_{3}\left(k_{i}\right)=3$ for $2 \leq i \leq 4$, since $e=r+1=3$, which implies $\frac{e-1}{2}=1$, and $G / G^{\prime \prime}$ has no defect of commutativity. The parameter $m$ is the index of nilpotency of $G / G^{\prime \prime}$, whence the nilpotency class is given by $c=m-1$.

Now, the statements are an immediate consequence of $\S \S 4.1-4.2$ in our recent article [2], where the claims are reduced to theorems in our earlier papers: [16] (Thm.1.3, p. 405), and, more generally, [18] (Thm.4.4, p.440 and Tbl.4.7, p. 441). We must only take into consideration that the 3-class group $\mathrm{Cl}_{3}(L)$ of $L$ is nearly homocyclic with abelian type invariants $A(3, c) \simeq(u+1, u)$, since $u=\frac{m-2}{2}$, and thus $2 u+1=m-1=c$.

Proof. (Proof of Theorem 6.1) First, we use the Artin pattern of $G$, as given in Corollary 6.1, to narrow down the possibilities for $G$. The possible class-3 quotients of $G$ are exactly the immediate descendants of the common class-2 quotient $\langle 27,3\rangle$ of all 3-groups with abelianization of type $(3,3)$ (apart from $\langle 27,4\rangle$ ), that is, four vertices $\langle 81,7 \ldots 10\rangle$ of step size 1 [1] (Figure 3 ), and seven vertices $\langle 243,3 \ldots 9\rangle$ of step size 2 [1] (Figure 4). All descendants of the former are of coclass 1 and reveal the same three stable (i.e. parameter independent) components $\left(\left(1^{2}\right)^{3}\right)$ of the first layer TTT $\tau_{1}$, according to [2] (Thm.3.2, (1)), which does not agree with the required TTT of $G$. Among the latter, the criterion [12] (Cor.3.0.2, p. 772) rejects three of the seven vertices, $\langle 243,3|4| 9\rangle$, since the TKT of $G$ does not contain a 2-cycle, and $\langle 243,5 \mid 7\rangle$ are discouraged, since they are terminal. The remaining two vertices $\langle 243,6 \mid 8\rangle$ are exactly the parents of the decisive groups $\langle 729,49 \mid 54\rangle$, where periodic bifurcations set in.

Now, Theorem 21.3 and Corollaries 21.2-21.3 in [1] (pp. 185-187) show that, using the local notation of Corollary 21.2,

$$
G \simeq S_{k}:=\langle 729,49 \mid 54\rangle(-\# 2 ; 1-\# 1 ; 1)^{k}-\# 2 ; 4|5| 6 \text { resp.2 }|4| 6
$$

and

$$
G / G^{\prime \prime} \simeq V_{0,2 k}:=\langle 729,49 \mid 54\rangle(-\# 1 ; 1)^{2 k}-\# 1 ; 4|5| 6 \text { resp.2| } 4 \mid 6,
$$

both with $k=u-2$.

\section{Computational Results for Three-Stage Towers}

With the aid of the computational algebra system MAGMA [11], where the class field theoretic techniques of Fieker [20] are implemented, we have determined the Artin pattern $(\tau(K) ; \varkappa(K))$ of all complex quadratic fields $K=\mathbb{Q}(\sqrt{d})$ with discriminants in the range $-10^{8}<d<0$, whose first layer TTT $\tau_{1}(K)$ had been 


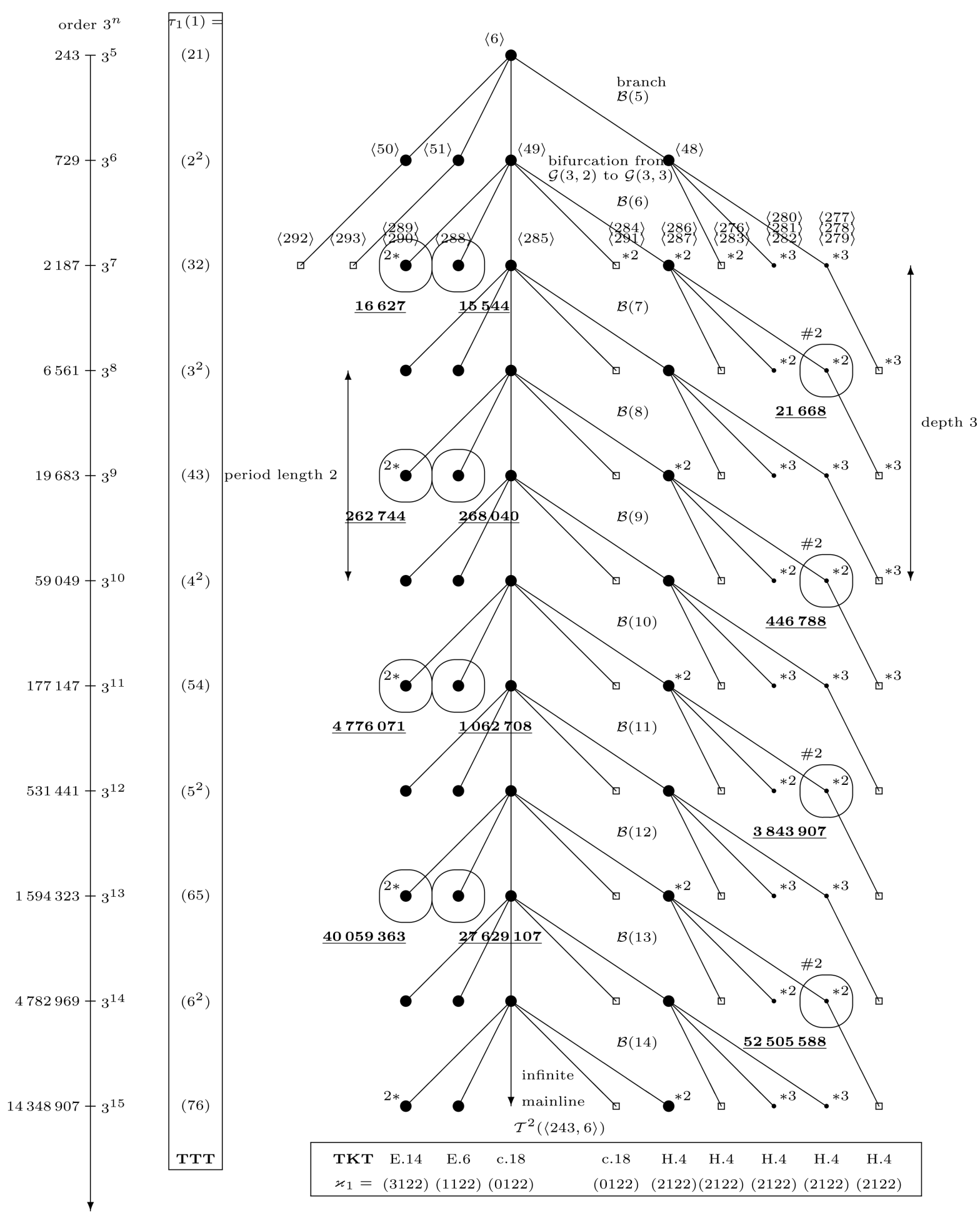

Figure 3. Minimal absolute discriminants $|d|<10^{8}$ distributed over $\mathcal{T}^{2}(\langle 243,6\rangle)$.

precomputed by Boston, Bush and Hajir in the database underlying the numerical results in [19].

Figure 3, resp. 4, shows the minimal absolute discriminant $|d|$, underlined and with boldface font, for which the adjacent vertex of the coclass tree $\mathcal{T}^{2}(\langle 729,49\rangle)$, resp. $\mathcal{T}^{2}(\langle 729,54\rangle)$, is realized as the metabe- 


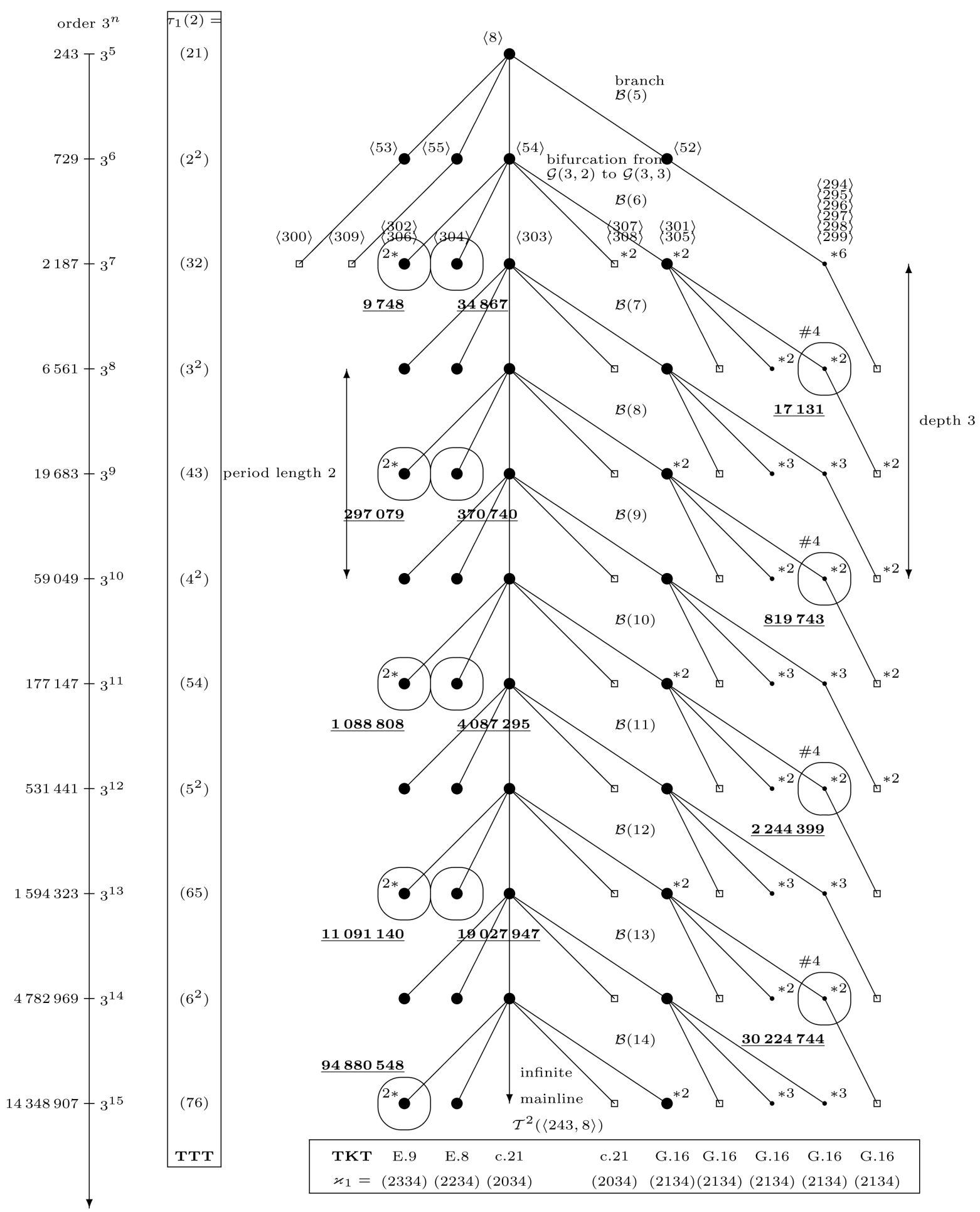

Figure 4. Minimal absolute discriminants $|d|<10^{8}$ distributed over $\mathcal{T}^{2}(\langle 243,8\rangle)$.

lianization $G / G^{\prime \prime}$ of the 3-tower group $G$ of $K=\mathbb{Q}(\sqrt{d})$. Vertices within the support of the distribution are surrounded by an oval. The corresponding projections $G \rightarrow G / G^{\prime \prime}$ have been visualized in the Figure 8 and Figure 9 of [1] (pp. 188-189). 
We have published this information in the Online Encyclopedia of Integer Sequences (OEIS) [21], sequences A247692 to A247697.

We emphasize that the results of section 6 provide the background for considerably stronger assertions than those made in [12]. Firstly, since they concern four TKTs E.6, E.14, E.8, E.9 instead of just TKT E.9 [2] (§4), and secondly, since they apply to varying odd nilpotency class $5 \leq \mathrm{cl}(G) \leq 19$ instead of just class 5 .

\section{Acknowledgements}

We gratefully acknowledge that our research is supported by the Austrian Science Fund (FWF): P 26008-N25. We are indebted to Nigel Boston, Michael R. Bush and Farshid Hajir for kindly making available an unpublished database containing numerical results of their paper [19].

\section{References}

[1] Mayer, D.C. (2015) Periodic Bifurcations in Descendant Trees of Finite p-Groups. Advances in Pure Mathematics, 5, 162-195. http://dx.doi.org/10.4236/apm.2015.54020 Special Issue on Group Theory, March 2015. (arXiv: 1502.03390v1 [math.GT] 11 Feb 2015.)

[2] Mayer, D.C. (2015) Index-p Abelianization data of p-Class Tower Groups, to appear in Advances in Pure Mathematics, 5, Special Issue on Number Theory and Cryptography, April 2015. (arXiv: 1502.03388v1 [math.NT] 11 Feb 2015.)

[3] Newman, M.F. (1977) Determination of Groups of Prime-Power Order. In: Lecture Notes in Math., vol. 573, Group Theory, Canberra, Springer, Berlin, 73-84. http://dx.doi.org/10.1007/bfb0087814

[4] O’Brien, E.A. (1990) The p-Group Generation Algorithm. Journal of Symbolic Computation, 9, 677-698. http://dx.doi.org/10.1016/S0747-7171(08)80082-X

[5] Besche, H.U., Eick, B. and O’Brien, E.A. (2002) A Millennium Project: Constructing Small Groups. Int. J. Algebra Comput., 12, 623-644. http://dx.doi.org/10.1142/S0218196702001115

[6] Besche, H.U., Eick, B. and O’Brien, E.A. (2005) The SmallGroups Library-A Library of Groups of Small Order. An accepted and refereed GAP 4 package, available also in MAGMA.

[7] Gamble, G., Nickel, W. and O’Brien, E.A. (2006) ANU p-Quotient-p-Quotient and p-Group Generation Algorithms. An accepted GAP 4 package, available also in MAGMA.

[8] The GAP Group (2015) GAP_-Groups, Algorithms, and Programming—a System for Computational Discrete Algebra. Version 4.7.7, Aachen, Braunschweig, Fort Collins, St. Andrews. http://www.gap-system.org

[9] Bosma, W., Cannon, J. and Playoust, C. (1997) The Magma Algebra System. I. The User Language. J. Symbolic Comput. 24, 235-265. http://dx.doi.org/10.1006/jsco.1996.0125

[10] Bosma, W., Cannon, J.J., Fieker, C. and Steels, A. (eds.) (2015) Handbook of Magma Functions. Edition 2.21, Univ. of Sydney, Sydney.

[11] The MAGMA Group (2015) MAGMA Computational Algebra System. Version 2.21-2, Sydney. http://magma.maths.usyd.edu.au

[12] Bush, M.R. and Mayer, D.C. (2015) 3-Class Field Towers of Exact Length 3. J. Number Theory, 147, 766-777. (arXiv: 1312.0251v1 [math.NT] 1 Dec 2013.)

[13] Hilbert, D. (1894) Ueber den Dirichlet'schen biquadratischen Zahlkörper. Mathematische Annalen, 45, 309-340. http://dx.doi.org/10.1007/BF01446682

[14] Azizi, A., Zekhnini, A. and Taous, M. (2015) Coclass of $\operatorname{Gal}\left(k_{2}^{(2)} \mid k\right)$ for Some Fields $k=\mathbb{Q}\left(\sqrt{p_{1} p_{2} q}, \sqrt{-1}\right)$ with 2-Class Groups of Type $(2,2,2)$. To appear in J. Algebra Appl.

[15] Artin, E. (1929) Idealklassen in Oberkörpern und allgemeines Reziprozitätsgesetz. Abh. Math. Sem. Univ. Hamburg, 7, 46-51. http://dx.doi.org/10.1007/BF02941159

[16] Mayer, D.C. (2013) The Distribution of Second p-Class Groups on Coclass Graphs. J. Théor. Nombres Bordeaux, 25, 401-456. (27th Journées Arithmétiques, Faculty of Mathematics and Informatics, Univ. of Vilnius, Lithuania, 2011.)

[17] Mayer, D.C. (2012) The Second p-Class Group of a Number Field. Int. J. Number Theory, 8, 471-505.

[18] Mayer, D.C. (2014) Principalization Algorithm via Class Group Structure. J. Théor. Nombres Bordeaux, 26, 415-464.

[19] Boston, N., Bush, M.R. and Hajir, F. (2015) Heuristics for p-Class Towers of Imaginary Quadratic Fields. To appear in Math. Annalen. (arXiv: 1111.4679v2 [math.NT] 10 Dec 2014.)

[20] Fieker, C. (2001) Computing Class Fields via the Artin Map. Math. Comp., 70, 1293-1303.

[21] Sloane, N.J.A. (2014) The On-Line Encyclopedia of Integer Sequences (OEIS). The OEIS Foundation Inc. http://oeis.org/ 